\title{
$Y$-meshes and generalized pentagram maps
}

\author{
Max Glick ${ }^{\dagger}$ and Pavlo Pylyavskyy ${ }^{\ddagger}$ \\ Department of Mathematics, University of Minnesota, Minneapolis, MN 55455, USA
}

\begin{abstract}
We introduce a rich family of generalizations of the pentagram map sharing the property that each generates an infinite configuration of points and lines with four points on each line. These systems all have a description as $Y$-mutations in a cluster algebra and hence establish new connections between cluster theory and projective geometry.

Résumé. Nous introduisons une famille de généralisations de l'application pentagramme. Chacune produit une configuration infinie de points et de lignes avec quatre points sur chaque ligne. Ces systèmes ont une description des $Y$-mutations dans une algèbre amassée, un nouveau lien entre la théorie d'algèbres amassées et la géométrie projective.
\end{abstract}

Keywords: pentagram map, discrete dynamical systems, cluster algebras

\section{Introduction}

The pentagram map, introduced by R. Schwartz [14] is a discrete dynamical system defined on the space of polygons in the projective plane. Figure 1(a) gives an example of the pentagram map, which we denote $T$. Although the underlying construction is simple, the resulting system has many amazing properties including the following:

- V. Ovsienko, Schwartz, and S. Tabachnikov [12] established that the pentagram map is a completely integrable system. Further work in this direction was conducted by those same authors [13], F. Soloviev [16], and M. Gekhtman, M. Shapiro, Tabachnikov, and A. Vainshtein [5].

- Schwartz [15] showed that the continuous limit of the pentagram map is the Boussineq equation.

- The first author [4] proved that the pentagram map can be described in certain coordinates as mutations in a cluster algebra.

The pentagram map has been generalized and modified in many ways with the hope that some or all of these properties continue to hold. Work of B. Khesin and Soloviev [6, 7, 8], Gekhtman, Shapiro, Tabachnikov and Vainshtein [5] and G. Mari Beffa [9, 10,11] all pursue this idea. Of the three listed properties, it seems that a connection to cluster algebras is the most resistant to being pushed forward, with only [5] achieving a generalization of the cluster description of $T$.

\footnotetext{
${ }^{\dagger}$ Partially supported by NSF grant DMS-1303482

‡Partially supported by NSF grants DMS-1068169, DMS-1148634, DMS-1351590, and Sloan Fellowship 


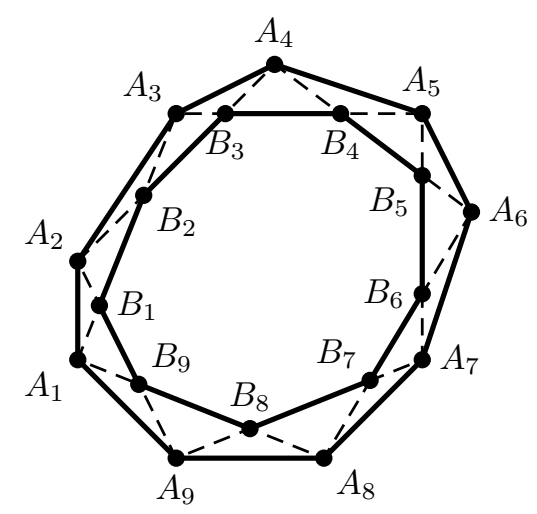

(a) An application of the pentagram map with input $A$ and output $B=T(A)$.

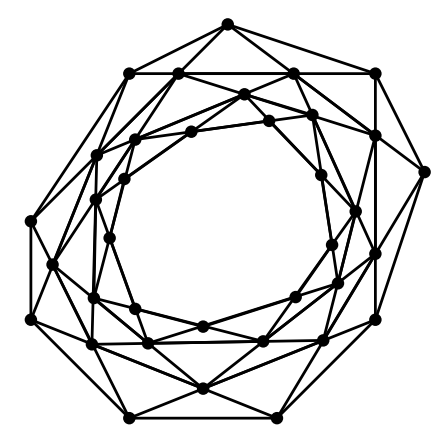

(b) Part of a $Y$-mesh produced by the pentagram map.

Fig. 1:

We propose a new family of generalized pentagram maps, all of which have a description in terms of cluster algebras. Our family includes the higher pentagram maps of Gekhtman et al. [5], some of those introduced by Khesin and Soloviev [6, 7], as well as a wide variety of new systems. Already for the systems of Khesin and Soloviev, the cluster structure is new.

For the pentagram map, the connection to cluster algebras comes by way of certain projectively natural coordinates on the space of polygons. Specifically, if $A$ is a polygon and $P_{i, j}$ is the $i$ th vertex of $T^{j}(A)$ (using the indexing method suggested by Figure 1(a)) then

$$
P_{i, j}, P_{i+2, j}, P_{i, j+1}, P_{i+1, j+1}
$$

are collinear for all $i, j \in \mathbb{Z}$ (see Figure 1(b)). The cross ratios of such quadruples play the role of $y$ variables in the associated cluster algebra. To obtain more systems admitting a cluster description, we reverse engineer the property of producing collinear points in a specified pattern.

Definition 1.1 Let $a, b, c, d \in \mathbb{Z}^{2}$ be distinct and assume $a_{2} \leq b_{2} \leq c_{2} \leq d_{2}$. Say that $S=\{a, b, c, d\}$ is a $Y$-pin if $b_{2}<c_{2}$ and the vectors $b-a, c-a, d-a$ generate all of $\mathbb{Z}^{2}$. When $S$ is $a Y$-pin, we will always assume its elements are called $a, b, c, d$ and that $a_{2} \leq b_{2} \leq c_{2} \leq d_{2}$.

Definition 1.2 Let $S=\{a, b, c, d\}$ be a $Y$-pin and suppose $D \geq 2$. A $Y$-mesh of type $S$ and dimension $D$ is a grid of points $P_{i, j}$ and lines $L_{i, j}$ in $\mathbb{R P}^{D}$ with $i, j \in \mathbb{Z}$ which together span all of $\mathbb{R P}^{D}$ and such that

- $P_{r+a}, P_{r+b}, P_{r+c}, P_{r+d}$ all lie on $L_{r}$ and are distinct for all $r \in \mathbb{Z}^{2}$

- $L_{r-a}, L_{r-b}, L_{r-c}, L_{r-d}\left(\right.$ all of which contain $\left.P_{r}\right)$ are distinct for all $r \in \mathbb{Z}^{2}$.

Note that the points determine the lines (for example, via $L_{r}=\left\langle P_{r+a}, P_{r+b}\right\rangle$ ) so we usually consider only the points. 
Given four points $x_{1}, x_{2}, x_{3}, x_{4} \in \mathbb{R P}^{1}=\mathbb{R} \cup\{\infty\}$, their cross ratio is defined to be

$$
\left[x_{1}, x_{2}, x_{3}, x_{4}\right]=\frac{\left(x_{1}-x_{2}\right)\left(x_{3}-x_{4}\right)}{\left(x_{2}-x_{3}\right)\left(x_{4}-x_{1}\right)} .
$$

The value of the cross ratio is that it is invariant under projective transformations. Given a $Y$-mesh $P=\left(P_{i, j}\right)_{i, j \in \mathbb{Z}}$ of type $S=\{a, b, c, d\}$, let

$$
y_{r}(P)=-\left[P_{r+a}, P_{r+c}, P_{r+b}, P_{r+d}\right]
$$

for all $r \in \mathbb{Z}^{2}$.

Theorem 1.3 Fix a $Y$-mesh of type $S=\{a, b, c, d\}$ and let $y_{r}=y_{r}(P)$. Then

$$
y_{r+a+b} y_{r+c+d}=\frac{\left(1+y_{r+a+c}\right)\left(1+y_{r+b+d}\right)}{\left(1+y_{r+a+d}^{-1}\right)\left(1+y_{r+b+c}^{-1}\right)}
$$

for all $r \in \mathbb{Z}^{2}$. Moreover, there exists a quiver $Q_{S}$ and a sequence of mutations so that the $y$-variables transform according to (1.2).

The second part of Theorem 1.3, namely the construction of the desired quiver, can be seen as an extension of work of A. Fordy and R. Marsh [3].

Theorem 1.3 ignores the fundamental issue of whether $Y$-meshes actually exist for given type $S$ and dimension $D$. In the case $S=\{(0,0),(2,0),(0,1),(1,1)\}$ and $D=2$, we have seen that such $Y$-meshes can be constructed by iterating the pentagram map. It is not hard to show for the same $S$ that $D=2$ is the only case that is realizable. Our main result is a description of which pairs $S$ and $D$ can be obtained, with an explicit definition of the corresponding pentagram like map in all realizable cases. First we need some definitions and notation.

Let $\mathcal{U}_{D}$ be the space of infinite polygons $A$ with vertices $\ldots, A_{-1}, A_{0}, A_{1}, A_{2}, \ldots$ in $\mathbb{R P}^{D}$, considered modulo projective equivalence. Let $\mathcal{U}_{D, m}$ denote the space of $m$-tuples $\left(A^{(1)}, \ldots, A^{(m)}\right)$ of polygons, also modulo projective equivalence. Let $\pi_{j}: \mathcal{U}_{D, m} \rightarrow \mathcal{U}_{D}$ denote the $j$ th projection map. For $A \in \mathcal{U}_{D, m}$, let $\langle A\rangle$ denote the span (i.e. affine hull) of the points of $A$.

Throughout we let $A^{(j)}$ denote the $j$ th row of a grid $\left(P_{i, j}\right)_{i, j \in \mathbb{Z}}$, that is $A_{i}^{(j)}=P_{i, j}$. We consider birational maps $F$ of $\mathcal{U}_{D, m}$ (or subvarieties thereof) of the form $F\left(A^{(1)}, \ldots, A^{(m)}\right)=\left(A^{(2)}, \ldots, A^{(m+1)}\right)$ and iterate $F$ and $F^{-1}$ to define $A^{(j)}$ for all $j \in \mathbb{Z}$.

Definition 1.4 Let $S=\{a, b, c, d\}$ be a $Y$-pin. Let $X \subseteq \mathcal{U}_{D, m}$ and assume $\langle A\rangle=\mathbb{R P}^{D}$ for generic $A \in X$. Say $F: X \rightarrow X$ as above is an $S$-map if for all $r$ and generic $A \in X$, the points $P_{r+a}, P_{r+b}, P_{r+c}, P_{r+d}$ are collinear and distinct. Call $m$ the order of the $S$-map and $D$ the dimension.

By way of notation, let $D(S)=2 \alpha-1$ where $\alpha$ is the area of the convex hull of $S$ (note $D(S)$ is an integer by Pick's formula).

Theorem 1.5 Let $S=\{a, b, c, d\}$ be a $Y$-pin.

1. If $2 \leq D \leq D(S)$ then there exists an $S$-map in dimension $D$ of order $m=d_{2}-a_{2}$.

2. If $D>D(S)$ then no $Y$-mesh of type $S$ exists in dimension $D$. 


\section{Definition of the $S$-maps}

\subsection{The $S$-map in the plane}

We begin by introducing the $S$-map in the plane, which will capture most of the essential components of the general definition.

Proposition 2.1 Let $S$ be a $Y$-pin with $D(S)>1$ and let $P$ be a $Y$-mesh of type $S$. Then

$$
P_{r+c+d}=\left\langle P_{r+a+c}, P_{r+b+c}\right\rangle \cap\left\langle P_{r+a+d}, P_{r+b+d}\right\rangle .
$$

and

for all $r \in \mathbb{Z}^{2}$.

$$
P_{r+a+b}=\left\langle P_{r+a+c}, P_{r+a+d}\right\rangle \cap\left\langle P_{r+b+c}, P_{r+b+d}\right\rangle
$$

Let $m=d_{2}-a_{2}$. We impose relations on $\mathcal{U}_{2, m}$ of two types (L1) and (L2) defined as follows:

- (L1) $P_{r+a}, P_{r+b}, P_{r+c}$ are collinear

- (L2) $P_{r+b}, P_{r+c}, P_{r+d}$ are collinear

Note that a relation like $P_{r+a}, P_{r+b}, P_{r+d}$ collinear would never fit within $m$ consecutive rows since $m=d_{2}-a_{2}$. Define $X_{2, S} \subseteq \mathcal{U}_{2, m}$ by all relevant (L1) and (L2) relations. Specifically, $A=$ $\left(A^{(1)}, \ldots, A^{(m)}\right) \in X_{2, S}$ if and only if (L1) holds for all $r$ with $-a_{2}<r_{2} \leq m-c_{2}$ and (L2) holds for all $r$ with $-b_{2}<r_{2} \leq m-d_{2}$. Recall here the identification $P_{i, j}=A_{i}^{(j)}$.

Define $A^{(m+1)} \in \overline{\mathcal{U}}_{2}$ using (2.1) (in the case $r_{2}=m+1-c_{2}-d_{2}$ ) and define $A^{(0)} \in \mathcal{U}_{2}$ using (2.2) (in the case $r_{2}=-a_{2}-b_{2}$ ). Write

$$
\begin{aligned}
& F(A)=\left(A^{(2)}, \ldots, A^{(m+1)}\right) \\
& G(A)=\left(A^{(0)}, \ldots, A^{(m-1)}\right)
\end{aligned}
$$

Proposition 2.2 Generically, $F(A) \in X_{2, S}$ and $G(A) \in X_{2, S}$. Moreover $F, G: X_{2, S} \rightarrow X_{2, S}$ are inverse as rational maps.

We now fix notation $T_{2, S}: X_{2, S} \rightarrow X_{2, S}$ for the map $F$ constructed above. Starting from $A \in X_{2, S}$, we can iterate $T_{2, S}$ and $T_{2, S}^{-1}$ to fill up the whole $P_{i, j}$ array, and all (L1) and (L2) relations will hold. It follows that generically $P_{r+a}, P_{r+b}, P_{r+c}$, and $P_{r+d}$ are collinear.

Example 2.3 Let $S=\{(-1,1),(1,2),(0,3),(0,4)\}$ and $D=2$. Figure 2 illustrates the $S$-map

$$
T_{2, S}: X_{2, S} \rightarrow X_{2, S}
$$

A triple of enclosed points indicates that said points are collinear (as are any triple in the same relative position). In this example, the (L1) relation is $P_{i-1,1}, P_{i+1,2}, P_{i, 3}$ collinear for all $i$ and the (L2) relation is $P_{i+1,1}, P_{i, 2}, P_{i, 3}$ collinear for all $i$. The space $X_{2, S} \subseteq \mathcal{U}_{2,3}$ consists of triples of infinite polygons satisfying these relations.

The two line segments indicate the construction of a $P_{i, 4}$, specifically the one marked by $a *$. The $S$-map $T_{2, S}$ is defined by

$$
P_{i, 4}=\left\langle P_{i-1,1}, P_{i+1,2}\right\rangle \cap\left\langle P_{i-1,2}, P_{i+1,3}\right\rangle \text {. }
$$




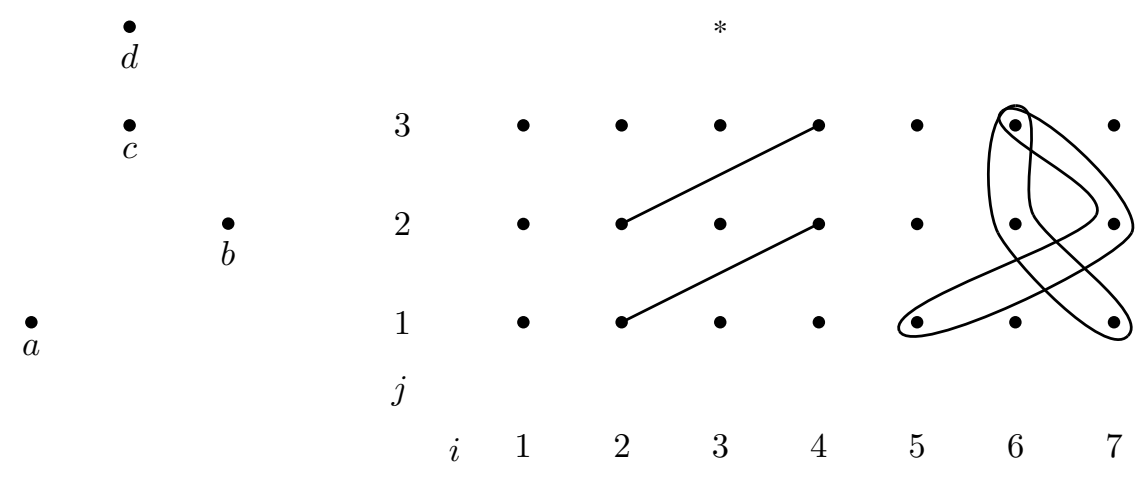

Fig. 2: The $Y$-pin $S=\{(-1,1),(1,2),(0,3),(0,4)\}$ (left) and the corresponding $S$-map (right).

\subsection{Higher dimensional S-maps}

Now suppose $D>2$, but keep $m=d_{2}-a_{2}$. The identities (2.1) and (2.2) still follow from the definition of a $Y$-mesh. The only new feature is a certain coplanarity condition (P3), which is needed in addition to (L1) and (L2) from before:

- (P3) $P_{r+a+c}, P_{r+a+d}, P_{r+b+c}, P_{r+b+d}$ are coplanar.

Define $X_{D, S} \subseteq \mathcal{U}_{D, m}$ by $A \in X_{D, S}$ if and only if the $P_{i, j}=A_{i}^{(j)}$ satisfy (L1), (L2), and (P3) for all relevant $r$. Specifically, (L1) should hold for $-a_{2}<r_{2} \leq m-c_{2}$, (L2) should hold for $-b_{2}<r_{2} \leq$ $m-d_{2}$, and (P3) should hold for $-a_{2}-c_{2}<r_{2} \leq m-b_{2}-d_{2}$.

The maps $F: X_{D, S} \rightarrow X_{D, S}$ and $G: X_{D, S} \rightarrow X_{D, S}$ are defined using (2.1) and (2.2) as before. By a (P3) relation, the four points used in the construction are coplanar making it possible to join them in pairs and intersect the resulting lines.

Proposition 2.4 The maps $F$ and $G$ do in fact map $X_{D, S}$ to $X_{D, S}$, and they are inverse to each other.

Let $T_{D, S}: X_{D, S} \rightarrow X_{D, S}$ denote the map $F$. Apply $T_{D, S}$ forwards and backwards to build the full $P_{i, j}$ array. It follows as usual from the (L1) and (L2) relations that

$$
P_{r+a}, P_{r+b}, P_{r+c}, P_{r+d}
$$

are collinear for all $r \in \mathbb{Z}^{2}$.

We seem to have obtained an $S$-map in all dimensions $D$. What is missing is the requirement that the resulting $Y$-meshes actually span all of $\mathbb{R P}^{D}$ rather than some proper subspace. Theorem 1.5 then boils down to being able to understand generic elements of $X_{D, S}$.

Proposition 2.5 If $D \leq D(S)$ then generic $A \in X_{D, S}$ span all of $\mathbb{R P}^{D}$. If $D>D(S)$ then every $A \in X_{D, S}$ actually lies in some $D(S)$ dimensional subspace. 


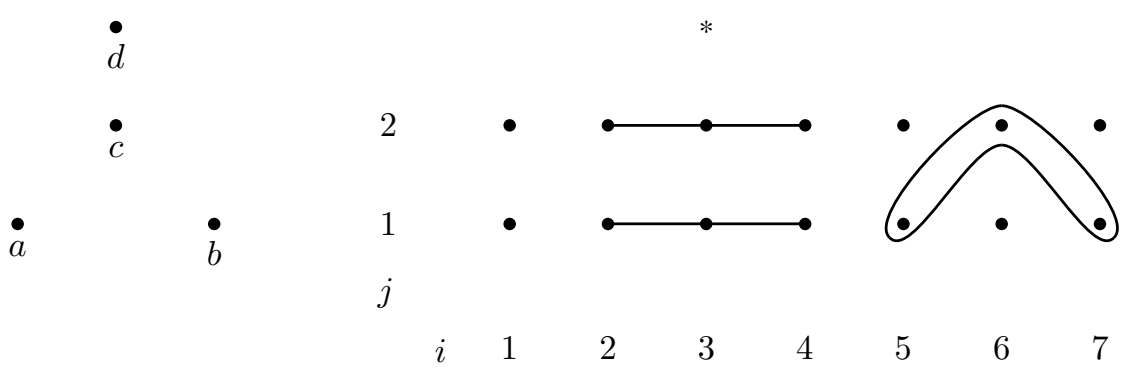

Fig. 3: The $S$-map for $S=\{(-1,1),(1,1),(0,2),(0,3)\}$.

\section{Connection with other pentagram maps}

Example 3.1 Fix $p$, q relatively prime with $1 \leq q<p$. Let $S=\{(0,0),(p, 0),(0,1),(q, 1)\}$ and $D=2$. Then $m=1-0=1$. The (L1) and (L2) relations do not fit, so arbitrary polygons are allowed, i.e. $X_{2, S}=\mathcal{U}_{2}$. Letting $A_{i}=P_{i, 1}$ and $B_{i}=P_{i, 2}$, equation (2.1) (applied at $r=(i-q, 0)$ ) implies $T_{2, S}(A)=B$ where

$$
B_{i}=\left\langle A_{i-q}, A_{i+p-q}\right\rangle \cap\left\langle A_{i}, A_{i+p}\right\rangle .
$$

In words, $B$ is formed by taking distance $p$ diagonals of $A$, and then intersecting diagonals that are $a$ distance $q$ apart. The case $p=2, q=1$ corresponds to the pentagram map.

Note the convex hull of $S$ is a trapezoid of area $(p+q) / 2$ so $D(S)=p+q-1$. If $2<D \leq p+q-1$ then $X_{D, S} \subseteq \mathcal{U}_{D}$ is defined by a (P3) relation

$$
X_{D, S}=\left\{A \in \mathcal{U}_{D}: A_{i}, A_{i+q}, A_{i+p}, A_{i+p+q} \text { coplanar for all } i\right\} .
$$

The map $T_{D, S}$ is defined by (3.1) as before. In the case $q=1$, Gekhtman, Shapiro, Tabachnikov, and Vainshtein [5] extensively studied the corresponding systems ( $T_{D, S}$ in our notation, $T_{p+1}$ in theirs). They call these maps higher pentagram maps and the polygons on which they act corrugated polygons.

Example 3.2 Let $S=\{(-1,1),(1,1),(0,2),(0,3)\}$ and $D=3$. Figure 3 illustrates the corresponding map. Letting $A_{i}=P_{i, 1}$ and $B_{i}=P_{i, 2}$, we have $(A, B) \in X_{3, S}$ if and only if $A_{i-1}, B_{i}, A_{i+1}$ collinear and $A_{i-1}, A_{i+1}, B_{i-1}, B_{i+1}$ coplanar for all $i$. The $S$-map is $T_{3, S}(A, B)=(B, C)$ where

$$
C_{i}=\left\langle A_{i-1}, A_{i+1}\right\rangle \cap\left\langle B_{i-1}, B_{i+1}\right\rangle .
$$

Surprisingly, the polygon $C$ can be determined from A alone via

$$
C_{i}=\left\langle A_{i-2}, A_{i}, A_{i+2}\right\rangle \cap\left\langle A_{i-1}, A_{i+1}\right\rangle,
$$

that is $C=F(A)$ for $F: \mathcal{U}_{3} \rightarrow \mathcal{U}_{3}$ the short diagonal hyperplane map of Khesin and Soloviev [6].

The map $F$ in the previous example is not itself an $S$-map. More precisely, it does not trace out a full $Y$-mesh, but rather every second row of a $Y$-mesh. A large family of $S$-maps are related in a similar way 
to so-called $(I, J)$-maps defined by Khesin and Soloviev [7]. Fix $D \geq 2$ and let $I=\left(s_{1}, \ldots, s_{D-1}\right)$, $J=\left(t_{1}, \ldots, t_{D-1}\right)$. Given $A \in \mathcal{U}_{D}$ define the $I$-hyperplanes of $A$ to be

$$
H_{i}=\left\langle A_{i}, A_{i+s_{1}}, A_{i+s_{1}+s_{2}}, \ldots, A_{i+s_{1}+\ldots+s_{d-1}}\right\rangle .
$$

Define a new polygon $B$ whose vertices are the $J$-intersections of the $H_{i}$

$$
B_{i-i_{0}}=H_{i} \cap H_{i+t_{1}} \cap H_{i+t_{1}+t_{2}} \cap \ldots \cap H_{i+t_{1}+\ldots+t_{d-1}} .
$$

Everything is defined up to some uniform shift $i_{0}$ of indices. The map $T_{I, J}: \mathcal{U}_{D} \rightarrow \mathcal{U}_{D}$ is $T_{I, J}(A)=B$.

A basic property of these maps is that they are birational. In fact, if $I=\left(s_{1}, \ldots, s_{D-1}\right), J=$ $\left(t_{1}, \ldots, t_{D-1}\right)$ then $T_{I, J}^{-1}=T_{J^{*}, I^{*}}$ where $I^{*}=\left(s_{D-1}, \ldots, s_{1}\right)$ and $J^{*}=\left(t_{D-1}, \ldots, t_{1}\right)$. We can always take the $s_{k}$ and $t_{k}$ to be positive, but it will be convenient to allow negative values as well. There is no problem with the definition provided the partial sums of $I$ are all distinct and similarly for $J$.

Say that a $Y$-pin $S$ is horizontal if $a_{2}=b_{2}$. Applying a translation and by choice of $a$ we can assume $a_{2}=b_{2}=0$ and $0=a_{1}<b_{1}$. Let $p=c_{2}$ and $q=d_{2}$. In order to have $b-a, c-a, d-a$ span all of $\mathbb{Z}^{2}$, it must be the case that $p, q$ are relatively prime.

Conjecture 3.3 Suppose $S$ is horizontal as above. Then the map $\pi_{1}: X_{p+q, S} \rightarrow \mathcal{U}_{p+q}$ is surjective. Restricting to twisted configurations, i.e. A satisfying $A_{i+n}^{(j)}=\phi\left(A_{i}^{(j)}\right)$ for some fixed $n \geq 1$ and projective transformation $\phi$, the map $\pi_{1}$ is finite-to-one.

Proposition 3.4 Suppose $S$ is horizontal as above. Let $\left(P_{i, j}\right)_{i, j \in \mathbb{Z}}$ be a $Y$-mesh of type $S$ and dimension $p+q$. As usual, let $A^{(j)}$ be the polygon $\ldots, P_{0, j}, P_{1, j} \ldots$.. Assuming Conjecture 3.3,

$$
A^{(j+p q)}=T_{I, J}\left(A^{(j)}\right)
$$

where $I=(s, s, \ldots, s), J=(s, \ldots, s, t, s, \ldots, s), s=b_{1}>0, t=q c_{1}-p d_{1}-(q-1) b_{1}$, and the unique $t$ of $J$ is in position $p$. Note for this statement to make sense we must add the assumption that the partial sums of $J$ are all distinct, which is true for instance whenever $t>0$.

In words, the map $T_{I, J}$ traces out every $p q$ th row of a $Y$-mesh. Hence the $S$-map $T_{p+q, S}$ can be thought of as a $p q$ th root of $T_{I, J}$, up to the conjecturally finite cover $\pi_{1}$. The process can be reversed and any $I, J$ as above can be realized, assuming $s, t$ are relatively prime and the position of the $t$ is relatively prime to $D$. Taking inverses we also get such pairs with $I$ and $J$ reversed.

Table 1 lists some $(I, J)$-maps in three dimensions along with the corresponding $S$. The list includes the short diagonal hyperplane map [6], the dented and deep dented maps [7], and an unnamed map investigated numerically in [8]. To fit the short diagonal map $I=(2,2), J=(1,1)$ in our framework, it is necessary to rewrite $J$ in the equivalent form $J=(-1,2)$. For these examples we can verify Conjecture 3.3. In general, we get many but not all of the systems that Khesin and Soloviev have proven to be integrable. For instance, if $D=4$ our setup cannot handle the dented map $I=(1,2,1), J=(1,1,1)$ because the dent is in position 2 which is not relatively prime to $D$.

\section{Decreasing the order}

We have focused on constructing $S$-maps of given type $S$ and dimension $D$, when possible. Rather than also determine the possible orders $m$ for the maps, we have simply fixed $m=d_{2}-a_{2}$, which works. It is natural to look for the smallest order possible, but this value is not known in general. In this section, we address the case $D=2$ proving that the minimal order is $m=\max \left(c_{2}-a_{2}, d_{2}-b_{2}\right)$. 


\begin{tabular}{l|l|l|l} 
Map name & $I$ & $J$ & $S$ \\
\hline Short diagonal & $(2,2)$ & $(1,1)$ & $\{(0,0),(2,0),(1,1),(1,2)\}$ \\
Dented & $(1,2)$ & $(1,1)$ & $\{(0,0),(1,0),(2,-1),(1,-2)\}$ \\
Deep dented & $(1, t)$ & $(1,1)$ & $\{(0,0),(1,0),(t,-1),(t-1,-2)\}$ \\
$?$ & $(2,2)$ & $(1,2)$ & $\{(0,0),(2,0),(2,1),(1,2)\}$
\end{tabular}

Tab. 1: The $Y$-pin $S$ associated with several $(I, J)$-maps.

Example 4.1 Recall Example 2.3 in which $S=\{(-1,1),(1,2),(0,3),(0,4)\}$ and $D=2$. Let $A_{i}=$ $P_{i, 1}, B_{i}=P_{i, 2}$, and $C_{i}=P_{i, 3}$ for all $i \in \mathbb{Z}$. Note for $(A, B, C) \in X_{2, S}$, the twisted polygons $A$ and $B$ can be arbitrary, and for a generic choice of such, $C$ is uniquely determined. Indeed, we must have

$$
C_{i}=\left\langle A_{i-1}, B_{i+1}\right\rangle \cap\left\langle A_{i+1}, B_{i}\right\rangle
$$

Hence $T_{2, S}$ has a smaller order analogue $F: \mathcal{U}_{2,2} \rightarrow \mathcal{U}_{2,2}$ given by $F(A, B)=(B, C)$ for $C$ as in (4.1).

The general construction depends on which of $c_{2}-a_{2}$ and $d_{2}-b_{2}$ is larger. We present the case $d_{2}-b_{2} \geq c_{2}-a_{2}$. For the remainder of the section, let $m^{\prime}=d_{2}-b_{2}$. Define a new space $X^{\prime} \subseteq \mathcal{U}_{2, m^{\prime}}$ by (L1) relations alone, that is $A \in X^{\prime}$ if and only if (L1) holds for all $r$ with $-a_{2}<r_{2} \leq m^{\prime}-c_{2}$.

Now for $A \in X^{\prime}$ define $F(A)=\left(A^{(2)}, \ldots, A^{\left(m^{\prime}+1\right)}\right)$ where $A^{\left(m^{\prime}+1\right)}$ is defined by

$$
P_{r+c+d}=\left\langle P_{r+2 c}, P_{r+b+c}\right\rangle \cap\left\langle P_{r+a+d}, P_{r+b+d}\right\rangle
$$

for $r \in \mathbb{Z}^{2}$ with $r_{2}=m^{\prime}+1-c_{2}-d_{2}$. Define $G(A)=\left(A^{(0)}, \ldots, A^{\left(m^{\prime}-1\right)}\right)$ where $A^{(0)}$ is defined by

$$
P_{r+a+b}=\left\langle P_{r+a+c}, P_{r+a+d}\right\rangle \cap\left\langle P_{r+b+c}, P_{r+2 b}\right\rangle .
$$

Proposition 4.2 For generic $A \in X^{\prime}, F(A)$ and $G(A)$ are also in $X^{\prime}$, and $F, G: X^{\prime} \rightarrow X^{\prime}$ are inverse as rational maps. Moreover, $F$ is an $S$-map of order $m^{\prime}$. Lastly, no $S$-map of smaller order exists for $D=2$.

Let $m_{D}(S)$ denote the minimal possible order of an $S$-map in dimension $D$. We have shown $m_{2}(S)=$ $\max \left(c_{2}-a_{2}, d_{2}-b_{2}\right)$. In general, it seems $m_{2}(S) \geq m_{3}(S) \geq \ldots \geq m_{D(S)}(S)$. We conclude this section with an example where $m_{3}(S)$ is strictly less than $m_{2}(S)$.

Example 4.3 Let $S=\{(0,1),(0,2),(-1,3),(1,5)\}$. By the results of this section, there is an order $\max (3-1,5-2)=3$ map $F: X \rightarrow X$ when $D=2$. The map is pictured in Figure 4 . The space $X \subseteq \mathcal{U}_{2,3}$ is defined by the relations

$$
P_{i, 1}, P_{i, 2}, P_{i-1,3}
$$

collinear, and $F$ is defined by

$$
P_{i, 4}=\left\langle P_{i-1,1}, P_{i-2,2}\right\rangle \cap\left\langle P_{i+1,2}, P_{i+1,3}\right\rangle .
$$

We claim when $D=3$ the order can be reduced to 2. Define $X^{\prime} \subseteq \mathcal{U}_{3,2}$ by requiring that the three subspaces (one line and two planes)

$$
\left\langle P_{i+1,1}, P_{i+1,2}\right\rangle,\left\langle P_{i-1,1}, P_{i-2,2}, P_{i-2,1}\right\rangle,\left\langle P_{i, 2}, P_{i-2,1}, P_{i-3,2}\right\rangle
$$




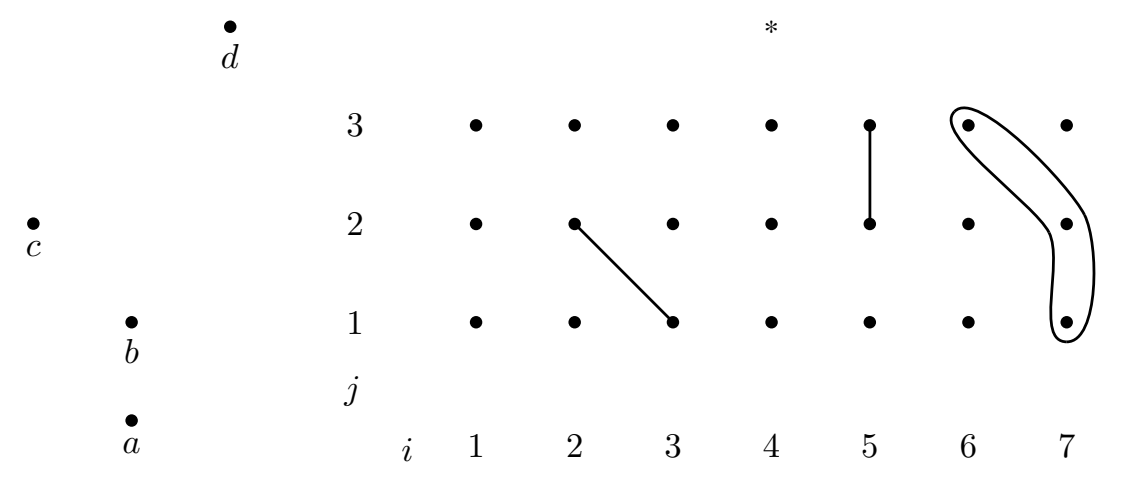

Fig. 4: The $S$-map for $S=\{(0,1),(0,2),(-1,3),(1,5)\}$.

pass through a common point, for all $i$. Let $P_{i, 3}$ be this common point, and define $G: X^{\prime} \rightarrow X^{\prime}$ accordingly. That the defining relation of $X^{\prime}$ is preserved by $G$ is on the surface a miracle, as is the fact that the system produces a $Y$-mesh. However, these results can be proven without much work at this point by connecting $G$ to the previously defined $S$-map.

\section{The cluster description of $S$-maps}

This section addresses the proof of Theorem 1.3. First, the identity (1.2) can be rewritten in the form "a product of six cross ratios equals one". Extracting a minimal set of points and lines needed reduces the identity to the following.

Lemma 5.1 Let $P_{1}, P_{2}, P_{3}, P_{4}$ be points and $L_{1}, L_{2}, L_{3}, L_{4}$ lines, all in general position in $\mathbb{R P}^{2}$. For $1 \leq i<k \leq 4$, let

$$
y_{i, k}=\left[P_{i},\left\langle P_{i}, P_{k}\right\rangle \cap L_{j}, P_{k},\left\langle P_{i}, P_{k}\right\rangle \cap L_{l}\right]
$$

where $\{j, l\}=\{1,2,3,4\} \backslash\{i, k\}$ are chosen so that $i, j, k, l$ is an even permutation. Then

$$
\prod_{1 \leq i<k \leq 4} y_{i, j}=1
$$

We now turn to the construction of the quiver, which requires reviewing definitions pertaining to cluster algebras. Cluster algebras, introduced by S. Fomin and A. Zelevinsky [1] are commutative rings endowed with certain combinatorial structure. We will restrict ourselves to the case when this extra structure can be encoded by a quiver. A quiver $Q$ is a directed graph without loops or oriented two-cycles.

Let $v$ be a vertex in a quiver $Q$. The quiver mutation $\mu_{v}$ transforms $Q$ into the new quiver $\mu_{v}(Q)$ defined as follows:

- For each pair of directed edges $u \longrightarrow v$ and $v \longrightarrow w$, add a new edge $u \longrightarrow w$.

- Reverse directions of all edges incident to $v$. 


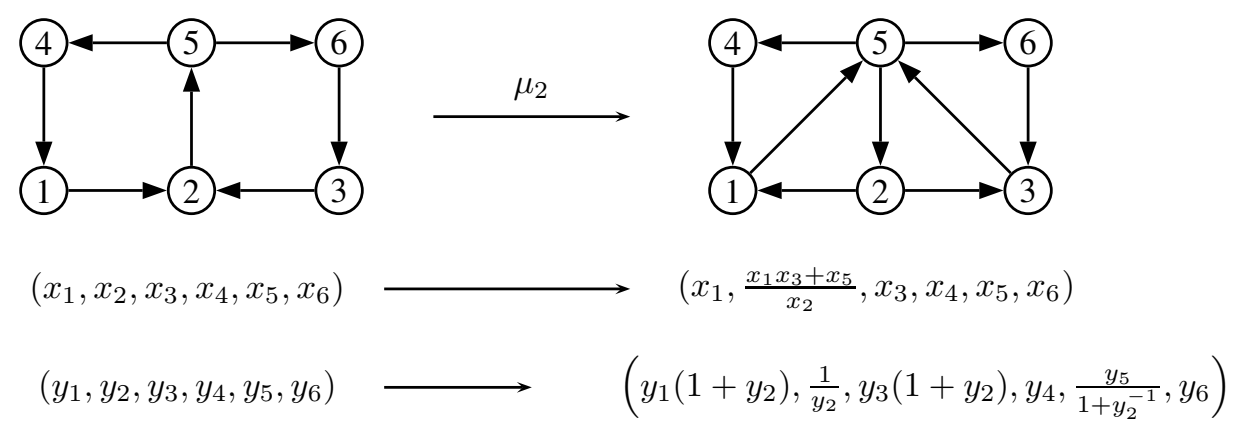

Fig. 5: An example of a quiver mutation, along with the corresponding birational maps of the $x$ and $y$ variables.

- Remove all oriented two-cycles.

A seed is a pair $(Q, \mathbf{x})$ where $\mathbf{x}=\left(x_{v}\right)_{v \in V}$ is indexed by the vertex set $V$ of $Q$. Given a vertex $v$, the seed mutation $\mu_{v}$ transforms $(Q, \mathbf{x})$ into the seed $\left(Q^{\prime}, \mathbf{x}^{\prime}\right)=\mu_{v}(Q, \mathbf{x})$ where $Q^{\prime}=\mu_{v}(Q)$ as defined above, $x_{u}^{\prime}=x_{u}$ whenever $u \neq v$, and

$$
x_{v} x_{v}^{\prime}=\prod_{v \longrightarrow w} x_{w}+\prod_{w \longrightarrow v} x_{w}
$$

There is an auxiliary notion of $Y$-seeds $(Q, \mathbf{y})$ with $\mathbf{y}=\left(y_{v}\right)_{v \in V}$ and an associated mutation rule [2]. Specifically, given a vertex $v, \mu_{v}$ transforms $(Q, \mathbf{y})$ to the $Y$-seed $\left(Q^{\prime}, \mathbf{y}^{\prime}\right)=\mu_{v}(Q, \mathbf{y})$ where $Q^{\prime}=\mu_{v}(Q)$ and

$$
y_{u}^{\prime}= \begin{cases}\left(y_{v}\right)^{-1} & u=v \\ y_{u}\left(\prod_{u \longrightarrow v}\left(1+y_{v}\right)\right)\left(\prod_{v \longrightarrow u}\left(1+y_{v}^{-1}\right)\right)^{-1}, & \text { otherwise }\end{cases}
$$

Figure 5 gives an example of seed and $Y$-seed mutations.

Definition 5.2 (Fordy and Marsh [3]) Say a quiver $Q$ on vertex set $V=\{1,2, \ldots, m\}$ has period one if $\mu_{1}(Q)=\rho(Q)$ where $\rho$ denotes the procedure of relabeling the vertices according to $\rho(j)=j+1$ for $1 \leq j<m$ and $\rho(m)=1$.

Begin with an initial seed $(\mathbf{x}, Q)$ with $\mathbf{x}=\left(x_{j}\right)_{j=1, \ldots, m}$ and $Q$ period one. Perform mutations in the following order: $\mu_{1}, \mu_{2}, \ldots, \mu_{m}, \mu_{1}, \mu_{2}, \ldots$. Call the single new variable produced by the $k$ th mutation in this sequence $x_{m+k}$. Then the periodicity of the quiver ensures

$$
x_{j+m} x_{j}=\prod_{1 \rightarrow k+1} x_{j+k}+\prod_{k+1 \rightarrow 1} x_{j+k}
$$

for all $j \in \mathbb{Z}$ where the products are over arrows in the original quiver $Q$.

Now suppose we consider a $Y$-seed with a period one quiver $Q$ and the same mutation sequence. There are more distinct $y$-variables because each one is potentially changed by each mutation. However, it is sufficient to keep track only of those variables that live at vertices that are about to be mutated. Call $y_{j}$ the variable at the vertex mutated at step $j$ for $j \in \mathbb{Z}$. 
Proposition 5.3

$$
y_{j+m} y_{j}=\frac{\prod_{(k+1) \rightarrow 1}\left(1+y_{j+m-k}\right)}{\prod_{1 \rightarrow(k+1)}\left(1+y_{j+m-k}^{-1}\right)}
$$

We now generalize the notion of period one quivers in order to model certain two dimensional recurrences. For this purpose, it is convenient to allow infinite quivers. We will require them however to be locally finite, meaning that each vertex is connected to only finitely many other vertices. Under this assumption, mutations can be defined as before. In fact, if $U$ is an infinite set of vertices no two of which are connected by an arrow, then we can define a simultaneous mutation $\mu_{U}$ at the vertices of $U$ thanks to the following property.

Proposition 5.4 Assume vertices $u$ and $v$ in $Q$ are not connected by an edge. Then the seed mutations (or $Y$-seed mutations) $\mu_{u}$ and $\mu_{v}$ commute.

Now fix vertex set $V=\mathbb{Z} \times\{1, \ldots, l\}$. Let $Q$ be a quiver on this set and let $\mu_{*, j}$ denote mutation at the set $\{(i, j): i \in \mathbb{Z}\}$ when defined. Say that $Q$ is period one if

- $Q$ is invariant under the translation $(i, j) \mapsto(i+1, j)$ of its vertices,

- no two vertices $\{(i, 1)\}$ are connected by an arrow, and

- $\mu_{1}(Q)=\rho(Q)$ where $\rho$ denotes the permutation of the vertices

$$
\rho(i, j)= \begin{cases}(i, j+1), & 1 \leq j<l \\ \left(i+i_{0}, 1\right), & j=l\end{cases}
$$

for some fixed shift $i_{0} \in \mathbb{Z}$.

Similarly to the one dimensional case, it is natural to consider the mutation sequence $\mu_{*, 1}, \ldots, \mu_{*, l}, \mu_{*, 1}, \ldots$ Given an initial seed $(Q, \mathbf{x})$ we can define $x_{i+i_{0}, j+l}$ to be the variable that $x_{i, j}$ transforms to during the $j$ th mutation in this sequence. We can define $y_{i, j}$ for $i, j \in \mathbb{Z}$ to be the $y$-variables at vertices about to be mutated in a similar way.

\section{Proposition 5.5}

$$
y_{u+\left(i_{0}, l\right)} y_{u}=\frac{\prod_{(0,1)+v \rightarrow(0,1)}\left(1+y_{u+\left(i_{0}, l\right)-v}\right)}{\prod_{(0,1) \rightarrow(0,1)+v}\left(1+y_{u+\left(i_{0}, l\right)-v}^{-1}\right)}
$$

In order to match (1.2) and (5.3), we need a period one quiver with prescribed arrows in and out of $(0,1)$. The following Proposition guarantees the existence of such a quiver, under certain conditions on these arrows which is satisfied in our case. The quiver corresponding to $S=\{(-1,1),(1,1),(0,2),(0,3)\}$ (see Example 3.2) is pictured in Figure 6.

Proposition 5.6 Let $m_{v}$ be a collection of integers indexed by $v \in \mathbb{Z}^{2}$ with $1 \leq v_{2} \leq l-1$ such that all but finitely many $m_{v}$ are zero and $m_{v}=m_{\left(i_{0}, l\right)-v}$ for all $v$. Then there exists a period one quiver $Q$ such that for all $v$, there are $\left|m_{v}\right|$ arrows from $(0,1)$ to $(0,1)+v$ (resp. from $(0,1)+v$ to $\left.(0,1)\right)$ if $m_{v} \geq 0$ (resp. $m_{v} \leq 0$ ). 


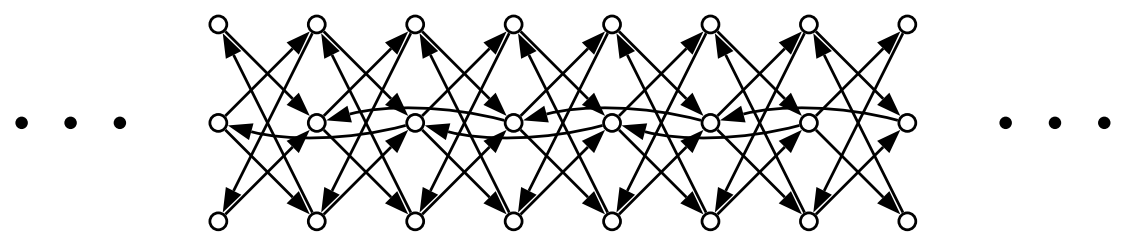

Fig. 6: The quiver $Q_{S}$ for $S=\{(-1,1),(1,1),(0,2),(0,3)\}$.

\section{References}

[1] S. Fomin and A. Zelevinsky, Cluster algebras I: Foundations, J. Amer. Math. Soc. 15 (2002), 497529.

[2] S. Fomin and A. Zelevinsky, Cluster algebras IV: Coefficients, Compos. Math. 143 (2007), 112-164.

[3] A. Fordy and R. Marsh, Cluster mutation-periodic quivers and associated Laurent sequences, $J$. Algebraic Combin. 34 (2011), 19-66.

[4] M. Glick, The pentagram map and Y-patterns, Adv. Math. 227 (2011), 1019-1045.

[5] M. Gekhtman, M. Shapiro, S. Tabachnikov, and A. Vainshtein, Higher pentagram maps, weighted directed networks, and cluster dynamics, Electron. Res. Announc. Math. Sci. 19 (2012), 1-17.

[6] B. Khesin and F. Soloviev, Integrability of higher pentagram maps, Math. Ann. 357 (2013), 100510047.

[7] B. Khesin and F. Soloviev, The geometry of dented pentagram maps, J. Eur. Math. Soc., to appear.

[8] B. Khesin and F. Soloviev, Non-integrability vs. integrability in pentagram maps, J. Geom. Phys. 87 (2015), 275-285.

[9] G. Mari Beffa, On generalizations of the pentagram map: discretizations of AGD flows, J. Nonlinear Sci. 23 (2013), 303-334.

[10] G. Mari Beffa, On integrable generalizations of the pentagram map, arxiv:1303.4295.

[11] G. Mari Beffa, On the integrability of the shift map on twisted pentagram spirals, arxiv:1406.6740.

[12] V. Ovsienko, R. Schwartz, and S. Tabachnikov, The pentagram map: a discrete integrable system, Comm. Math. Phys. 299 (2010), 409-446.

[13] V. Ovsienko, R. Schwartz, and S. Tabachnikov, Liouville-Arnold integrability of the pentagram map on closed polygons, Duke Math. J. 162 (2013), 2149-2196.

[14] R. Schwartz, The pentagram map, Experiment. Math. 1 (1992), 71-81.

[15] R. Schwartz, Discrete monodromy, pentagrams, and the method of condensation, J. Fixed Point Theory Appl. 3 (2008), 379-409.

[16] F. Soloviev, Integrability of the Pentagram Map, Duke Math. J. 162 (2013), 2815-2853. 